\title{
EQUILIBRIUM OF PERMANENT MULTIVALUED SYSTEMS
}

\author{
BY \\ LI YONG, WANG HUAIZHONG, AND LÜ XIANRUI \\ Department of Mathematics, Jilin University, People's Republic of China
}

\begin{abstract}
This paper is concerned with the existence problem of an equilibrium state for permanent multivalued systems. It is proved that the permanence of multivalued systems implies the existence of an equilibrium state by using the approximating technique of set-valued maps and asymptotic fixed point theory.
\end{abstract}

1. Introduction. Recently, there have been many works on permanence for some biological systems based on the following model system :

$$
x_{i}^{\prime}=x_{i} f_{i}(x), \quad i=1,2, \ldots, n,
$$

on the positive cone $R_{+}^{n}=\left\{x=\left(x_{1}, x_{2}, \ldots, x_{n}\right): x_{i} \geq 0\right.$, for $\left.i=1,2, \ldots, n\right\}$ (see [1, 2, 5, 7-13]). Here system (1) is said to be permanent if there exist $m$, $M \in(0, \infty)$ such that, given $x \in \operatorname{int} R_{+}^{n}$, there is a $t_{x}$ such that

$$
m \leq x_{i}(t) \leq M, \quad i=1,2, \ldots, n, t \geq t_{x} .
$$

From a biological point of view, it is reasonable to expect that, if permanence holds, there will be a stationary coexistence state in int $R_{+}^{n}$. For this problem, an affirmative answer was given by Hofbauer and Sigmund [8], Hutson [10], and Hutson and Moran [11]. Particularly in [10], Hutson provided a very simple proof with the use of some asymptotic fixed point theorems of Schauder and Horn. There he also raised the following open problem:

For a system of differential inclusion

$$
x_{i}^{\prime} \in F_{i}(x), \quad i=1,2, \ldots, n,
$$

where the $F_{i}$ 's are set-valued maps, does the permanence of the system imply the existence of an equilibrium state?

In the present paper, we shall give a positive answer for the above problem. Because in general we do not know whether the Poincare map of solutions to system (2) is convex set valued, a direct application of Hutson's method seems to be very difficult. To overcome this difficulty, we treat Eq. (2) as an approximate ordinary differential equation, utilizing the technique of Zaremba [16].

Received December 9, 1991.

1991 Mathematics Subject Classification. Primary 34A60.

(C)1993 Brown University 
2. Preliminaries. Let us recall some basic notation, definitions, and facts.

In the sequel, $K\left(R^{n}\right)$ denotes the set of all nonempty compact subsets of $R^{n}$ with the Hausdorff metric $\rho$ defined by

$$
\rho(A, B)=\max \{\beta(A, B), \beta(B, A)\}, \quad A, B \in K\left(R^{n}\right)
$$

where

$$
\beta(A, B)=\sup \{\operatorname{dist}(x, B): x \in A\} .
$$

Hence, with metric $\rho, K\left(R^{n}\right)$ is a complete metric space. Let

$$
K v\left(R^{n}\right)=\left\{A \in K\left(R^{n}\right): A \text { is convex }\right\} .
$$

A function $g: R^{m} \rightarrow K\left(R^{n}\right)$ is said to be upper semicontinuous (u.s.c.) at $p \in R^{m}$, if for each $\varepsilon>0$ there exists a $\delta>0$ such that $\beta(g(q), g(p))<\varepsilon$ whenever $q \in R^{m},|q-p|<\delta . g$ is u.s.c. on $R^{m}$ if it is u.s.c. at each point of $R^{m}$. We say $g$ is continuous, if it is continuous as a map into $K\left(R^{n}\right)$.

A function $x:[a, b] \rightarrow R^{n}$ is said to be a solution of system (2), if it is absolutely continuous and satisfies (2) almost everywhere on $[a, b]$. If $x(t)$ is a solution of system (2) defined on $R$ and for some constant $\omega>0, x(t+\omega)=x(t)$, for $t \in R$, then $x(t)$ is said to be an $\omega$-periodic solution of system (2).

The following lemmas are vital to our discussions.

Lemma 1 (Zaremba, [16]). Let $D \in K v\left(R \times R^{n}\right)$ and $F: D \rightarrow K v\left(R^{n}\right)$ be u.s.c. Then there exists a sequence $\left\{F_{k}\right\}$ of continuous functions from $D$ to $K v\left(R^{n}\right)$ possessing the following properties:

(i) for each $k$ and all $(t, x) \in D, F_{k+1}(t, x) \subset F_{k}(t, x)$;

(ii) for each $k$ and all $(t, x) \in D, F(t, x) \subset$ int $F_{k}(t, x)$;

(iii) for each $(t, x) \in D$ and $\varepsilon>0$, there exist $\delta, N>0$ such that $F_{k}(s, y) \subset$ $S_{\varepsilon}(F(t, x)) \equiv\{(u, v) \in D: \operatorname{dist}((u, v), F(t, x))<\varepsilon\}$ for $(s, y) \in S_{\delta}(t, x)$ and $k \geq N$.

Lemma 2 (Kelley, [15]). Let $D \in K v\left(R \times R^{n}\right)$ and $F: D \rightarrow K v\left(R^{n}\right)$ and $F_{k}: D \rightarrow$ $K v\left(R^{n}\right) \quad(k=1,2, \ldots)$ satisfy (i)-(iii) of Lemma 1. Suppose moreover that there are an interval $[a, b]$ and a sequence $\left\{x_{k}(t)\right\}$ such that, for each $k, x_{k}(t)$ is a solution of $x^{\prime} \in F_{k}(t, x)$ on $[a, b]$ and $\left(t, x_{k}(t)\right) \in D$. Then there exists a subsequence of $x_{k}(t)$ converging uniformly to a solution $x(t)$ of $x^{\prime} \in F_{k}(t, x)$ on $[a, b]$.

Lemma 3 (Filippov, [3]). Let $F: R^{m} \rightarrow K v\left(R^{n}\right)$ be continuous and $P \in R^{n}$. Then the function $f: R^{m} \rightarrow R^{n}$ defined by

$$
f(x) \in F(x), \quad|f(x)-P|=\operatorname{dist}\{F(x), P\} \quad \text { for } x \in R^{m}
$$

is continuous.

LemMA 4 (Filippov, [4, Theorem 1, p. 87]). Let $F: G \subset R \times R^{n} \rightarrow K v\left(R^{n}\right)$ be u.s.c., where $G$ is an open region; let $t_{0} \in[a, b],\left(t_{0}, x_{0}\right) \in G$, and $a, b \in R$. Suppose each solution $x(t)$ of the Cauchy problem

$$
x^{\prime} \in F(t, x), \quad x\left(t_{0}\right)=x_{0}
$$


is extensible to $[a, b]$ and $(t, x(t)) \in G$. Then for each $\varepsilon>0$, there exists a $\delta>0$ such that each solution of the Cauchy problem

$$
x^{\prime} \in F^{*}(t, x), \quad x\left(t_{0}^{*}\right)=x_{0}^{*}
$$

is extensible to $[a, b]$ and differs from some solution of Eq. (3) by not more than $\varepsilon$, for $t_{0}^{*} \in[a, b], x_{0}^{*} \in R^{n}$ and each upper semicontinuous function $F^{*}: G \rightarrow K v\left(R^{n}\right)$ with

$$
\begin{gathered}
\left|t_{0}^{*}-t_{0}\right| \leq \delta, \quad\left|x_{0}^{*}-x_{0}\right| \leq \delta, \\
F^{*}(t, x) \subset \bar{S}_{\delta}[\operatorname{CO}(\bigcup\{F(s, y):|s-t| \leq \delta, \quad|y-x| \leq \delta\})] .
\end{gathered}
$$

Lemma 5 (Jones, [14]). Let $S, S_{0}, S_{1}$ be convex subsets of a Fréchet space $X$ such that $S_{0} \subset S_{1} \subset S$, where $S$ is closed, $S_{1}$ open with respect to $S$, and $S_{0}$ compact; and let $T$ be a continuous map of $S$ into $X$. Suppose that $\overline{T\left(S_{1}\right)}$ is locally compact and there exists a positive integer $m$ such that $T^{i}$ is well defined on $S_{1}$ for $i=1,2, \ldots, m$ and $T^{m}\left(S_{1}\right) \subset S_{0}, T^{m+1}\left(S_{1}\right) \subset S_{0}$. Then $T$ has a fixed point in $S_{0}$.

3. Main result. We shall prove the following.

THEOREM. Let $F: R^{n} \rightarrow K v\left(R^{n}\right)$ be u.s.c. If system (2) is permanent, then an equilibrium state of (2) exists on int $R_{+}^{n}$, i.e., there exists an $x^{*} \in$ int $R_{+}^{n}$ such that $0 \in F\left(x^{*}\right)$.

Proof. Since system (2) is permanent, there exist constants $m_{0}, M>0$ such that for each $x_{0} \in$ int $R_{+}^{n}$ there exists a $t_{x_{0}} \in R_{+}$such that each solution $x(t)$ of the Cauchy problem

$$
x^{\prime} \in F(x), \quad x(0)=x_{0}
$$

satisfies

$$
m_{0} \leq x_{i}(t) \leq M, \quad i=1,2, \ldots, n, \quad t \geq t_{x_{0}} .
$$

By the finite covering theorem, (5), and Lemma 4, there exist constants $L, T>0$ such that for $x_{0} \in R^{n}$ with $m_{0} \leq x_{0_{i}} \leq M$ each solution $x(t)$ of (4) satisfies

$$
\begin{aligned}
& \left|x_{i}(t)\right| \leq L, \quad i=1,2, \ldots, n, \quad t \in[0, T], \\
& m_{0} \leq x_{i}(t) \leq M, \quad i=1,2, \ldots, n, \quad t \in[T, T+2] .
\end{aligned}
$$

For each $r, s>1$, set $D_{r}=\left\{x \in R^{n}:\left|x_{i}\right| \leq L+r\right\}, S_{s, r}=\left\{x \in R_{+}^{n}: m_{0}(1-1 / s r) \leq\right.$ $\left.x_{i} \leq M+1 / s r\right\}$. Applying Lemma 1 , we get a sequence $\left\{F_{k}(x)\right\}$ of continuous functions from $D_{2}$ to $R^{n}$ with properties (i)-(iii) in Lemma 1 , where $D=R^{+} \times D_{2}$ and $F(t, x)=F(x)$. By virtue of Lemma 3, there exists a sequence $\left\{f_{k}(x)\right\}$ of continuous functions such that

$$
f_{k}(x) \in F_{k}(x) \quad \text { for } x \in D_{2}, k=1,2, \ldots
$$

Hence by (6) and Lemma 4, for large $k$, each solution $x(t)$ of the Cauchy problem

$$
x^{\prime} \in F_{k}(x), \quad x(0)=x_{0}, \quad x_{0} \in S_{s, 2},
$$


satisfies

$$
x(t) \in D_{1}, \quad t \in[0, T] \quad \text { and } \quad x(t) \in S_{s, 4}, \quad t \in[T, T+2] .
$$

Particularly by (7), each solution $x(t)$ of the Cauchy problem

$$
x^{\prime}=f_{k}(x), \quad x(0)=x_{0}, \quad x_{0} \in S_{s, 4},
$$

satisfies (8).

For each $k$, choose a sequence of $C^{1}$ class functions $\left\{f_{k, m}\right\}$ on $D_{2}$ such that

$$
\lim _{m \rightarrow \infty} f_{k, m}(x)=f_{k}(x)
$$

uniformly on $D_{2}$. By (8) and the usual Kamke-like theorem (see Hartman [6, Chapter 2, Theorem 3.2]), for large $k$ and $m$, the solution $x_{k, m}\left(t, x_{0}\right)$ of the Cauchy problem

$$
x^{\prime}=f_{k, m}(x), \quad x(0)=x_{0}, \quad x_{0} \in S_{s, 2},
$$

satisfies

$$
x_{k, m}(t) \in D_{2}, \quad t \in[0, T], \quad \text { and } \quad x_{k, m}(t) \in S_{s, 3}, \quad t \in[T, T+2] .
$$

Take an $h_{0} \in(0,1)$ such that $3 h_{0} \leq 1$. For large $k, m$, and each $h \in\left(0, h_{0}\right)$, define a map $P_{k, m, h}: S_{s, 2} \rightarrow R^{n}$ by

$$
P_{k, m, h}\left(x_{0}\right)=x_{k, m}\left(h, x_{0}\right) \text {. }
$$

Since $f_{k, m}$ is of class $C^{1}, P_{k, m, h}$ is well defined. From (10) and the choice of $h_{0}$ we see that for large $k, m$, and each $h \in\left(0, h_{0}\right]$, there exists an integer $N=$ $N(k, m, h)>0$ such that $j h \in[T, T+2]$ and

$$
x_{k, m}\left(j h, x_{0}\right) \in S_{s, 3} \quad \text { for } x_{0} \in S_{s, 2}, j=N, N+1 \text {. }
$$

Since equation $x^{\prime}=f_{k, m}(x)$ is autonomous, we have

$$
P_{k, m, h}^{j}\left(x_{0}\right)=x_{k, m}\left(j h, x_{0}\right)
$$

for each integer $j \geq 0$. From (11) it follows that

$$
P_{k, m, h}^{j}\left(S_{s, 2}\right) \subset S_{s, 3} \subset S_{s, 2} \subset R^{n} \quad \text { for } j=N, N+1 .
$$

By this and Lemma $5, P_{k, m, h}$ has at least one fixed point $x_{k, m}^{*} \in S_{s, 3}$ i.e., $x_{k, m}\left(h, x_{k, m}^{*}\right)=x_{k, m}^{*}$. From the uniqueness of solutions to the Cauchy problem and the autonomous property of $f_{k, m}$ it follows that

$$
x_{k, m}\left(h+t, x_{k, m}^{*}\right)=x_{k, m}\left(t, x_{k, m}^{*}\right) \quad \text { for } k \in R \text {. }
$$

This shows that for any $h \in\left(0, h_{0}\right]$ the equation $x^{\prime}=f_{k, m}(x)$ has at least one $h$-periodic solution $x_{k, m}\left(t, x_{k, m}^{*}\right) \in D_{2}$ for $t \in R$. By virtue of Lemma 2 in [10], there exists a $y_{k, m} \in S_{s, 3}$ such that

$$
0=f_{k, m}\left(y_{k, m}\right)
$$


Hence by (9) and (12) there exists a $y_{k} \in S_{s, 3}$ such that

$$
0=f_{k}\left(y_{k}\right) \text {. }
$$

Note that $f_{k}(x) \in F_{k}(x)$ for $x \in D_{2}$. Therefore, (13) implies

$$
0 \in F_{k}\left(y_{k}\right) \text {; }
$$

i.e., $y_{k}$ is an equilibrium solution of $x^{\prime} \in F_{k}(x)$. Then by Lemma 2 , there exists an $x_{s}^{*} \in S_{s, 3}$ such that $0 \in F\left(x_{s}^{*}\right)$. The same argument as above implies that there is an $x^{*} \in R^{n}$ with $m_{0} \leq x_{i}^{*} \leq M, i=1,2, \ldots, n$, such that $0 \in F\left(x^{*}\right)$; i.e., $x^{*}$ an equilibrium state of $(2)$. The proof is complete.

\section{REFERENCES}

[1] T. Burton and V. Hutson, Repellers in systems with infinite delay, J. Math. Anal. Appl. 137, 240-263 (1989)

[2] G. Butler, H. Freedman, and P. Waltman, Uniformly persistent systems, Proc. Amer. Math. Soc. 96, 425-430 (1967)

[3] A. F. Filippov, Classical solutions of differential equations with multivalued right-hand sides, SIAM J. Control 5, 609-621 (1967)

[4] A. F. Filippov, Differential equations with discontinuous right-hand sides, Math. Appl., Kluwer, Dordrecht, 1988

[5] H. Freedman and J. So, Persistence in discrete semi-dynamical systems, SIAM J. Math. Anal. 20, 930-938 (1989)

[6] P. Hartman, Ordinary Differential Equations, Birkhäuser, Boston, MA, 1982

[7] J. Hofbauer, V. Hutson, and W. Jansen, Coexistence for systems governed by difference equations of Lotka-Volterra type, J. Math. Biol. 25, 553-570 (1987)

[8] J. Hofbauer and K. Sigmund, Dynamical systems and the theory of evolution, Cambridge Univ. Press, London and New York, 1988

[9] V. Hutson, A theorem on average Liapunov functions, Monatsh. Math. 98 4, 267-275 (1984)

[10] V. Hutson, The existence of an equilibrium for permanent systems, Rocky Mountain J. Math. 20, 1033-1040 (1990)

[11] V. Hutson and W. Moran, Persistence of species obeying difference equations, Math. Biosci. 15, 203-213 (1982)

[12] V. Hutson and W. Moran, Repellers in reaction-diffusion systems, Rocky Mountain J. Math. 17, 301-314 (1987)

[13] V. Hutson and J. Pym, Repellers for generalized semidynamical systems, Mathematics of Dynamic Processes (A. Kurzhanski, ed.), Lecture Notes in Econ. and Math. Systems, 287, Springer-Verlag, Berlin, 1987, pp. 39-49

[14] G. S. Jones, Asymptotic fixed point theory, Technical Notes BN-502, University of Maryland, College Park, 1967

[15] W. G. Kelley, Periodic solutions of generalized differential equations, SIAM J. Appl. Math. 30, 70-74 (1976)

[16] S. Zaremba, Sur les équations au paratingent, Bull. Sci. Math. 60, 139-160 (1936) 